\title{
Elevated Lipase Associated With COVID-19 Possibly due to Acute Pancreatitis in a Pediatric Patient: A Case Report
}

\author{
Shruthi Shekara, Neelam Sarwar ${ }^{\mathrm{a}, \mathrm{c}}$, Mudit Kalia ${ }^{\mathrm{a}}$, Joseph Castellanos ${ }^{\mathrm{a}}$, \\ Frances Pelliccia ${ }^{\mathrm{a}}$, Jagdip Gill ${ }^{\mathrm{b}}$
}

\begin{abstract}
As the global pandemic of severe acute respiratory syndrome coronavirus 2 (SARS-CoV-2) advances, the challenge of understanding its wide spectrum of symptoms and sequelae continues to be a priority in the medical world. There are not many case reports on the atypical presenting features of coronavirus disease 2019 (COVID-19), especially in the pediatric population. Discussed here is a case of acute pancreatitis in a 14-year-old male who presented with abdominal pain without any other typical COVID-19 symptoms. Workup was significant for positive SARS-CoV-2 polymerase chain reaction (PCR), elevated lipase, and computed tomography of the abdomen and pelvis with oral and intravenous contrast showed an indistinct margin of the pancreatic tail and left upper quadrant ascites, supportive of early pancreatitis. Clinical and laboratory improvement was noted after treatment with bowel rest, intravenous fluids, and antibiotics. This case highlights the concern that acute pancreatitis may be a complication associated with COVID-19. Identifying its signs may enable earlier diagnosis and treatment.
\end{abstract}

Keywords: Lipase; COVID-19; Acute pancreatitis; SARS-CoV-2

\section{Introduction}

Since being declared a pandemic, coronavirus disease 2019 (COVID-19) has become the subject of intense study in understanding how it presents clinically in the pediatric population [1]. In one case surveillance study of 12,689 children aged 10 - 19 years, fever and cough were reported in $35 \%$ and $41 \%$, respectively [2]. Abdominal pain and diarrhea were reported in

Manuscript submitted August 23, 2020, accepted October 9, 2020

Published online November 3, 2020

aGeneral Pediatrics Department, Children's Hospital at Saint Peter's University Hospital, New Brunswick, NJ 08901, USA

bPediatrics Emergency Department, Pediatrics Department, Children's Hospital at Saint Peter's University Hospital, New Brunswick, NJ 08901, USA

${ }^{\mathrm{c} C}$ Corresponding Author: Neelam Sarwar, General Pediatrics Department, Pediatrics Department, Children's Hospital at Saint Peter's University Hospital, New Brunswick, NJ 08901, USA. Email: nsarwar58@gmail.com

doi: https://doi.org/10.14740/ijcp401 approximately $8 \%$ and $14 \%$, respectively [2]. Gastrointestinal symptoms may occur without any respiratory symptoms [2, 3].

Acute pancreatitis in children is typically caused by viral infections like mumps, coxsackie, influenza, and Epstein-Barr virus (EBV). Severe acute respiratory syndrome coronavirus 2 (SARS-CoV-2) is not one of the recognized viruses [4]. The other etiologies of pancreatitis in children include drug abuse, biliary anomalies, gallstones, cholecystitis, trauma, metabolic diseases like diabetes and hypertriglyceridemia, familial and systemic diseases such as systemic lupus and juvenile rheumatoid arthritis [5]. Acute pancreatitis has been reported in adults and children affected with COVID-19 [6]. Amongst the pediatric population there are isolated case reports of acute pancreatitis and it is speculated as one of the gastrointestinal complications of COVID-19 [7, 8]. The following case highlights the importance of considering atypical manifestations of COVID-19 while evaluating gastrointestinal symptoms in a pediatric patient during this pandemic and seeks to add acute pancreatitis as one of the possible complications.

\section{Case Report}

A 14-year-old adolescent male presented to the emergency room with a 1-day history of abdominal pain that gradually worsened in severity. He described the pain as diffuse yet more prominent in his left upper quadrant. It was crampy in nature and associated with nausea, several bouts of non-bloody nonbilious emesis, and a decreased appetite.

He otherwise denied fevers, headaches, diplopia, conjunctivitis, anosmia, ageusia, cough, chest pain, dyspnea, diarrhea, rashes or trauma. The family denied any known COVID-19 exposure; however, both parents are nurses and state that they were screened weekly for COVID-19 via nasopharyngeal polymerase chain reaction (PCR) swab at their place of employment and all tests had been negative.

There was no significant past medical history. He denied any past surgeries, use of medications or known allergies. He denied using alcohol, tobacco, vaping or other illicit drugs. He was up to date on his vaccines. He otherwise had been developing appropriately for age, had a regular diet, and was living in a stable household.

Admission vitals were temperature of $36.8^{\circ} \mathrm{C}$, heart rate of 75 beats/minute, respiratory rate of 18 breaths $/ \mathrm{min}$, blood pressure of $121 / 85 \mathrm{~mm} \mathrm{Hg}$ and oxygen saturation of $98 \%$ on 
Table 1. Complete Blood Count

\begin{tabular}{|c|c|c|c|}
\hline & Hospital day 1 & Hospital day 2 & Hospital day 3 \\
\hline $\mathrm{WBC}\left(\times 10^{3} / \mathrm{mm}^{3}\right)$ & 9.8 & 9 & 9.4 \\
\hline $\mathrm{RBC}\left(\times 10^{6} / \mathrm{mm}^{3}\right)$ & 5.31 & 5.08 & 5.05 \\
\hline $\mathrm{HCT}(\%)$ & 43.6 & 42.1 & 41.9 \\
\hline MCV (fL) & 82.1 & 83 & 83 \\
\hline $\mathrm{MCHC}(\mathrm{g} / \mathrm{dL})$ & 33.8 & 33.2 & 33.1 \\
\hline MPV (fL) & 9.1 & 9.1 & 9 \\
\hline RDW-CV (\%) & 14.5 & 14.5 & 14.1 \\
\hline Platelet count $\left(\times 10^{3} / \mathrm{mm}^{3}\right)$ & 204 & 195 & 193 \\
\hline Neutrophils $(\%)$ & 84 & 76.4 & 79.7 \\
\hline Basophils (\%) & 0.0 & 0.4 & 0.5 \\
\hline Absolute neutrophils $\left(\times 10^{3} / \mathrm{mm}^{3}\right)$ & 8.23 & 6.9 & 7.5 \\
\hline Absolute lymphocytes $\left(\times 10^{3} / \mathrm{mm}^{3}\right)$ & 1.08 & 1.2 & 0.9 \\
\hline Absolute monocytes $\left(\times 10^{3} / \mathrm{mm}^{3}\right)$ & 0.39 & 0.9 & 0.9 \\
\hline Absolute eosinophils $\left(\times 10^{3} / \mathrm{mm}^{3}\right)$ & 0.1 & 0.0 & 0.0 \\
\hline Absolute basophils $\left(\times 10^{3} / \mathrm{mm}^{3}\right)$ & 0.0 & 0.0 & 0.0 \\
\hline
\end{tabular}

WBC: white blood cell; RBC: red blood cell; Hb: hemoglobin; HCT: hematocrit; MCV: mean corpuscular volume; MCH: mean corpuscular hemoglobin; $\mathrm{MCHC}$ : mean corpuscular hemoglobin concentration; MPV: mean platelet volume; RDW-CV: red blood cell distribution width.

room air. His physical exam was significant for a grade II/VI systolic murmur heard at the lower sternal border which increased in intensity when the patient was lying flat. On abdominal exam the patient had left sided abdominal tenderness more prominent in the left upper quadrant. The neurological exam was unremarkable with a Glasgow Coma Scale of 15, good strength, and tone. No signs of respiratory distress or dehydration.

The patient's labs were significant for an elevated neutrophil count of $84 \%$, elevated total bilirubin to $1.6 \mathrm{mg} / \mathrm{dL}$, lipase to 2,908 U/L, and positive SARS-CoV-2 nasopharyngeal PCR. He had a normal white blood cell (WBC) count, electrolytes, C-reactive protein (CRP), hepatic enzymes and triglyceride level. Urinalysis was initially indicative of dehydration but corrected with fluid replacement. Urine drug screen was negative. His workup was also negative for gonorrhea/chlamydia, human immunodeficiency virus (HIV) and syphilis (Tables 1-4). Viral panel to exclude other viral causes was deferred because of a shortage of PCR swabs and as per hospital policy at that time.

A limited ultrasound right lower abdomen was done to rule out acute appendicitis which showed a normal appendix. A computed tomography of the abdomen and pelvis with oral and intravenous contrast was ordered to rule out any other intra-abdominal pathology given his marked left sided abdominal tenderness on physical examination. It showed an indistinct margin of the pancreatic tail and left upper quadrant ascites. It was further discussed with a pediatric radiologist who noted subtle stranding at the tail of the pancreas. These findings were suggestive of early pancreatitis. There were no findings of gallstones or biliary tract obstruction (Fig. 1).

Patient was started on bowel rest, intravenous (IV) fluids, and pain management. On day 1 of admission, he received IV Tylenol 1,000 mg (total one dose) then switched to IV Toradol $30 \mathrm{mg}$ every $8 \mathrm{~h}$ (total seven doses) with Hydromorphone $1 \mathrm{mg}$ for breakthrough pain (total two doses). On day 2, he became febrile (101.4 F) and was started on IV Zosyn 3.375 g every $6 \mathrm{~h}$ (total two doses) and oral Metronidazole $500 \mathrm{mg}$ every $8 \mathrm{~h}$ (total two doses). He was also given oral famotidine $20 \mathrm{mg}$ twice daily for gastrointestinal prophylaxis (total three doses) and Zofran $4 \mathrm{mg}$ (total one dose) during hospital stay. Once clinical exams improved and lipase down trended, the diet was advanced and pain medication was switched to oral acetaminophen $650 \mathrm{mg}$ every $4 \mathrm{~h}$ as needed (total two doses). He tolerated advances well and remained stable on room air. His CRP level increased to $104 \mathrm{mg} / \mathrm{dL}$. He otherwise remained well and afebrile for over $24 \mathrm{~h}$ and lipase down trended to 148 $\mathrm{U} / \mathrm{L}$ prior to discharge. No antibiotics were prescribed upon discharge to home from the hospital. His hospital stay was approximately 3.5 days.

Of note, electrocardiogram (EKG) done during hospitalization which reported mild left ventricular hypertrophy 
Table 2. Complete Metabolic Panel

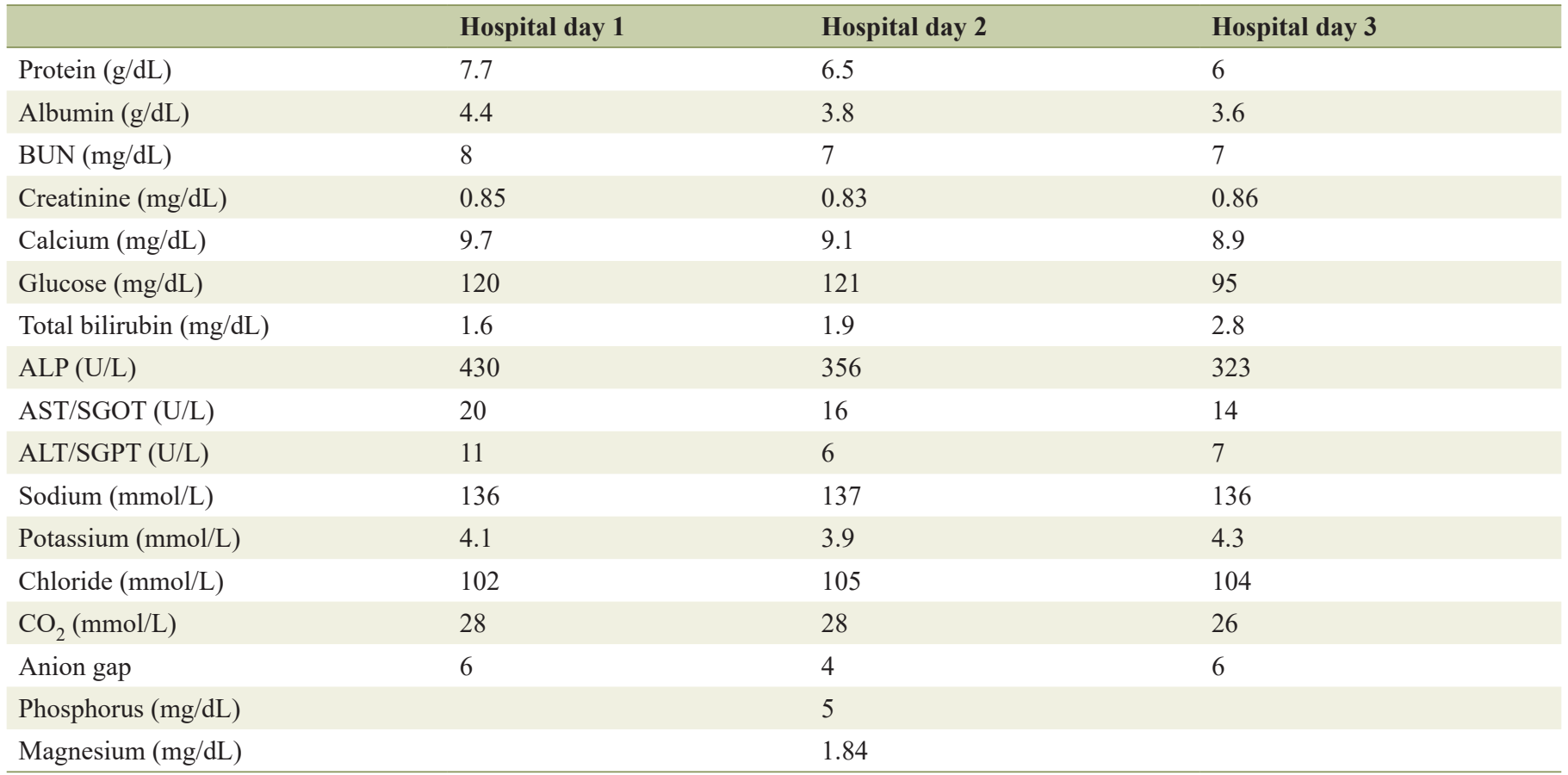

BUN: blood urea nitrogen; ALP: alkaline phosphatase; AST/SGOT: aspartate aminotransferase; ALT/SGPT: alanine aminotransferase; $\mathrm{CO}_{2}$ : carbon dioxide.

for evaluation of murmur. Cardiology advised follow-up in 2 weeks for evaluation of the murmur. Blood pressures noted to be in the $90-95 \%$ for age for which family was instructed to keep a log then follow up with a nephrologist as an outpatient visit. The patient, however, was lost to follow-up.

\section{Discussion}

In summary, this was a 14-year-old previously healthy adolescent male who presented with abdominal pain and was diagnosed with acute pancreatitis, likely secondary to SARS$\mathrm{CoV}-2$ virus. As a viral panel was not performed we cannot exclude co-infection with another virus such as mumps, coxsackie, influenza, or EBV that are associated with acute pancreatitis although patient did not manifest with any other symptoms or show lab results typical to these viruses. His hos- pital stay remained uneventful. Clinical and laboratory studies showed notable improvement with bowel rest, hydration, pain control and antibiotics. This case is unique due to its isolated gastrointestinal manifestations in the setting of a positive SARS-CoV-2 PCR test. Patient's diagnosis of COVID-19 is elucidated as the possible cause of his acute pancreatitis. Early recognition and management started in our emergency room contributed to the faster recovery of the patient.

Not much is known about the interaction of SARS-CoV-2 with the various organ systems, especially in the pediatric population $[9,10]$. Although there have been isolated case reports in the adult population and a retrospective/observational study from Wuhan Tongji Hospital and Wuhan Jinyintan Hospital in China about pancreatic injury caused by the COVID-19, the exact mechanism is still under investigation $[11,12]$. A plausible mechanism suggested is the binding of the SARS-CoV-2 to angiotensin-converting enzyme 2 (ACE2) in the pancreas

Table 3. Additional Serum Studies

\begin{tabular}{ll}
\hline Gamma-glutamyl transferase & $15 \mathrm{U} / \mathrm{L}$ \\
Amylase & $104 \mathrm{U} / \mathrm{L}$ \\
Triglycerides & $43 \mathrm{mg} / \mathrm{dL}$ \\
HIV $1 / 2$ antibody 4th generation screening & Non-reactive \\
RPR for syphilis & Non-reactive \\
Blood culture & Negative \\
Urine Chlamydia trachomatis and Neisseria gonorrhoeae & Negative \\
\hline
\end{tabular}

HIV: human immunodeficiency virus; RPR: rapid plasma reagin. 
Table 4. Urinalysis

\begin{tabular}{lll}
\hline & Hospital day $\mathbf{1}$ & Hospital day 2 \\
\hline Glucose & Negative & Negative \\
Blood & Negative & Negative \\
Bilirubin & Small & Negative \\
Ketones & Trace & Negative \\
Urine specific gravity & $\geq 1.030$ & $\geq 1.031$ \\
PH & 6.0 & 5.0 \\
Protein (mg/dL) & 100 & 30 \\
Urobilinogen (EU/dL) & 0.2 & $<2.0$ \\
Leukocyte esterase & Negative & Negative \\
Nitrites & Negative & Negative \\
WBC & & $2-5 / \mathrm{HPF}$ \\
Bacteria & & Few \\
Mucus threads & & $3+/ \mathrm{LPF}$ \\
\hline
\end{tabular}

WBC: white blood cell; HPF: high power field; LPF: low power field.

since the expression of this receptor is higher in the pancreas than the lungs [12].

Current literature focuses more on the respiratory presentation of COVID-19 [9, 13]. Gastrointestinal manifestations continue to be an unexplored dimension in the pediatric population $[3,14]$. There is no published literature on the management of pancreatic inflammation which might be attributed to the SARS-CoV-2, although the mainstay for acute pancreatitis management is fluids, pain control, antibiotics for secondary bacterial infection with initial bowel rest and early initiation of oral feeding [5]. The significant improvement in our patient with the above-mentioned regimen may serve as a guide to patient management with similar presenting features.

\section{Conclusions}

To our knowledge, there have been limited reported cases amongst the pediatric population with acute pancreatitis as the sole presenting feature after being infected with COVID-19. This case illustrates the vulnerability of the pediatric population to this virus and adds to the myriad of presenting symptoms that may be attributed to the novel SARS-CoV-2. A limitation of this case report is that a respiratory viral panel was not performed to exclude other viral etiologies associated with acute pancreatitis. This case does emphasize the need to consider acute pancreatitis as a differential for pediatric patients presenting only with abdominal pain during this pandemic and mandatory COVID-19 testing. Further research is required to explore this association and investigate the possible mechanism of injury and why this injury is more pronounced in certain organ systems.

\section{Acknowledgments}

None to declare.

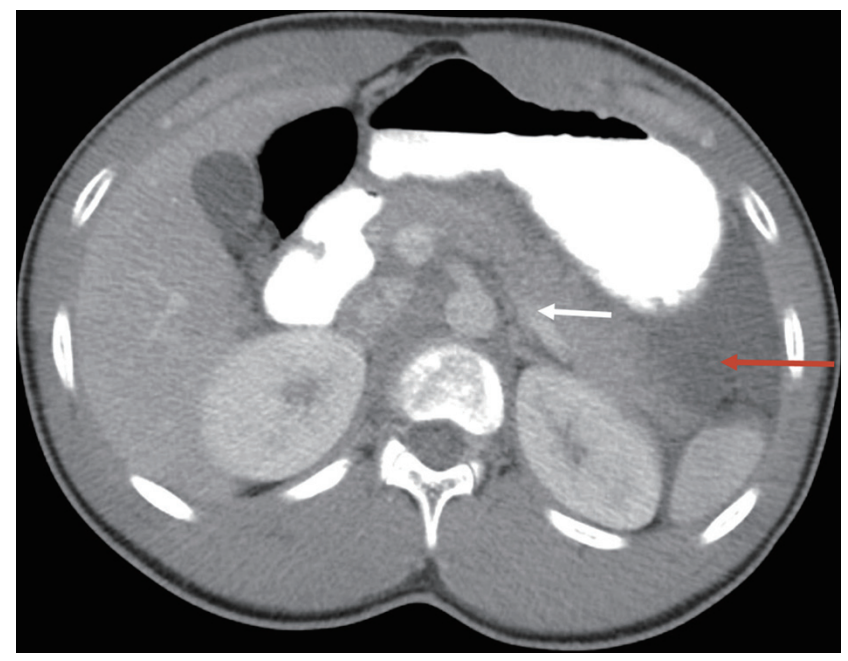

Figure 1. Computed tomography of the abdomen and pelvis showing an indistinct margin of the pancreatic tail and left upper quadrant ascites. Mild stranding (white arrow) with surrounding ascites (red arrow).

\section{Financial Disclosure}

None to declare.

\section{Conflict of Interest}

None to declare.

\section{Informed Consent}

Not applicable.

\section{Author Contributions}

SS, NS, MK, and JC drafted the initial manuscript. FP reviewed the manuscript. JG reviewed and revised the manuscript. All the authors have approved the final manuscript as submitted and accepted accountability for all aspects of the work.

\section{Data Availability}

The data supporting the findings of this study are available from the corresponding author upon reasonable request.

\section{References}

1. CDC COVID-19 Response Team. Coronavirus Disease 2019 in Children - United States, February 12-April 2, 2020. MMWR Morb Mortal Wkly Rep. 2020;69(14):422426.

2. Mustafa NM, L AS. Characterisation of COVID-19 Pan- 
demic in Paediatric Age Group: A Systematic Review and Meta-Analysis. J Clin Virol. 2020;128:104395.

3. Mao R, Qiu Y, He JS, Tan JY, Li XH, Liang J, Shen J, et al. Manifestations and prognosis of gastrointestinal and liver involvement in patients with COVID-19: a systematic review and meta-analysis. Lancet Gastroenterol Hepatol. 2020;5(7):667-678.

4. Suzuki M, Sai JK, Shimizu T. Acute pancreatitis in children and adolescents. World J Gastrointest Pathophysiol. 2014;5(4):416-426.

5. Pohl JF, Uc A. Paediatric pancreatitis. Curr Opin Gastroenterol. 2015;31(5):380-386.

6. https://www.ncbi.nlm.nih.gov/sites/myncbi/14SJefTqKq hQV/collections/60046662/public/.

7. Stevens JP, Brownell JN, Freeman AJ, Bashaw H. COVID-19-associated multisystem inflammatory syndrome in children presenting as acute pancreatitis. J Pediatr Gastroenterol Nutr. 2020.

8. Alloway BC, Yaeger SK, Mazzaccaro RJ, Villalobos T, Hardy SG. Suspected case of COVID-19-associated pancreatitis in a child. Radiol Case Rep. 2020;15(8):13091312.
9. Zimmermann P, Curtis N. Coronavirus Infections in Children Including COVID-19: an overview of the epidemiology, clinical features, diagnosis, treatment and prevention options in children. Pediatr Infect Dis J. 2020;39(5):355368.

10. Chang TH, Wu JL, Chang LY. Clinical characteristics and diagnostic challenges of pediatric COVID-19: A systematic review and meta-analysis. J Formos Med Assoc. 2020;119(5):982-989.

11. Bian XW. The COVID-19 Pathology Team. Autopsy of COVID-19 victims in China. Natl Sci Rev. 2020;7(9):1414-1418.

12. Liu Furong, Long Xin, Zou Wenbin, Fang Minghao, Wu Wenjuan, Li Wei, Zhang Bixiang, etal. Highly ACE2 expression in pancreas may cause pancreas damage after SARSCoV-2 infection. medRxiv. 2020:2020.02.28.20029181.

13. Dong Y, Mo X, Hu Y, Qi X, Jiang F, Jiang Z, Tong S. Epidemiology of COVID-19 among children in China. Pediatrics. 2020;145(6):e20200702.

14. Yang L, Tu L. Implications of gastrointestinal manifestations of COVID-19. Lancet Gastroenterol Hepatol. 2020;5(7):629-630. 